\title{
Genetic Algorithms used for Search and Rescue of Vulnerable People in an Urban Setting
}

\author{
Shuhad Aljandeel \\ sa0041@mix.wvu.edu
}

Follow this and additional works at: https://researchrepository.wvu.edu/etd

Part of the Other Applied Mathematics Commons

\section{Recommended Citation}

Aljandeel, Shuhad, "Genetic Algorithms used for Search and Rescue of Vulnerable People in an Urban Setting" (2020). Graduate Theses, Dissertations, and Problem Reports. 7596.

https://researchrepository.wvu.edu/etd/7596

This Thesis is protected by copyright and/or related rights. It has been brought to you by the The Research Repository @ WVU with permission from the rights-holder(s). You are free to use this Thesis in any way that is permitted by the copyright and related rights legislation that applies to your use. For other uses you must obtain permission from the rights-holder(s) directly, unless additional rights are indicated by a Creative Commons license in the record and/ or on the work itself. This Thesis has been accepted for inclusion in WVU Graduate Theses, Dissertations, and Problem Reports collection by an authorized administrator of The Research Repository @ WVU. For more information, please contact researchrepository@mail.wvu.edu. 
Graduate Theses, Dissertations, and Problem Reports

2020

Genetic Algorithms used for Search and Rescue of Vulnerable

People in an Urban Setting

SHUHAD ALJANDEEL

Follow this and additional works at: https://researchrepository.wvu.edu/etd

Part of the Other Applied Mathematics Commons 


\title{
Genetic Algorithms used for Search and Rescue of Vulnerable People in an Urban Setting
}

\author{
Shuhad Aljandeel
}

\author{
Thesis Submitted \\ to the Eberly College of Arts and Science \\ at West Virginia University \\ in partial fulfillment of the requirements for the degree of \\ Master in \\ Mathematics / Applied Mathematics \\ Marjorie Darrah, ph.D.,Chair \\ Harvey Diamond, ph.D. \\ Jessica Deshler, ph.D. \\ Department of Mathematics \\ Mrogantown, West Virginia \\ 2020
}

Keywords: Search and Rescue, GA, UAV

Copyright 2020 Shuhad Aljandeel 


\begin{abstract}
Genetic Algorithms used for Search and Rescue of Vulnerable People in an Urban Setting

Shuhad Aljandeel
\end{abstract}

The main goal of this research is to design and develop a genetic algorithm (GA) for path planning of an Unmanned Aerial Vehicle (UAV) outfitted with a camera to efficiently search for a lost person in an area of interest. The research focuses on scenarios where the lost person is from a vulnerable population, such as someone suffering from Alzheimer or a small child who has wondered off. To solve this problem, a GA for path planning was designed and implemented in Matlab. The area of interest is considered to be a circle that encompasses the distance the person could have walked in the time they have been missing. The area might also have some subareas that could not be excluded from the search for various reasons, such as a river they could not cross, or a fenced area. A grid is imposed on the area of interest, based on the field of view of the camera that the UAV is carrying and the height the UAV is flying. A chromosome is the encoding of the path the UAV will fly and the fitness function of the GA is designed to ensure that the UAV is covering all areas of the grid with the least amount of backtracking. The results show that the GA can find a path that efficiently covers the area. These results can be generalized to use more than one UAV. 


\section{ACKNOWLEDGMENT}

I would like to thank my supervisor professor Marjorie Darrah for her kindness and cooperation. There have been many situations that require her help and cooperation, she dedicated her time and efforts to help me finishing my thesis. I would also like to thank professors Harvey Diamond and Jessica Deshler for their patience during the defense of my thesis and for their notes and directions. I would like to thanks Billy William Tobin for his efforts and support helping me writing the MATLAB codes. I would like to thank Ryan Hansen for his support and patience editing the LATEX documents and directing me through the research printing. 


\section{Contents}

1 Introduction 1

1.1 History of Search and Rescue . . . . . . . . . . . . . . 1

1.2 Unmanned Aerial Vehicles . . . . . . . . . . . . . . . . 2

1.3 Unmanned Aerial Vehicle for SAR . . . . . . . . . . 3

1.4 Genetic Algorithms for Optimization . . . . . . . . . . . 4

$\begin{array}{llr}2 & \text { Research Methods } & 7\end{array}$

2.1 Scenario of Interest . . . . . . . . . . . . . . . . . . 7

2.2 Determining Search Area . . . . . . . . . . . . . . . . 8

2.3 Creating the GA for Path Planning . . . . . . . . . . 10

2.3.1 Fitness Function ............... 13

2.3.2 Crossover, Mutation, and Elitism . . . . . . . . 14

$\begin{array}{llr}3 & \text { Results } & 15\end{array}$

4 Conclusion and Future Work 25

$\begin{array}{llr}5 & \text { Appendix } & 29\end{array}$

5.1 Code for GA . . . . . . . . . . . . . . . . . . . 29

5.2 Crossover Code . . . . . . . . . . . . . . . . . . 31 


\section{Introduction}

\subsection{History of Search and Rescue}

It is well known that search and rescue (SAR) missions started with man a long time ago. More than 5000 years ago, people used different types of dogs to find missing people. For example, shepherd dogs, sight dogs, guard dogs and trailing dogs were used in those missions. As early as in the 1700 s, the monks at St.Bernard Hospice in Switzerland utilized SAR techniques by using many types of dogs. Some dogs found lost or dead people during missions between the mountains. The SAR techniques were widely used during the First World War. Dogs were trained by the French and Germans to find the missing live or dead soldiers. Moreover, during the Second World War the importance of dogs in SAR technique had increased where the British used dogs to locate the enemies positions. Also, the dogs were trained and used by Americans during the Korean and Vietnam war as message carriers [1].

Another type of SAR is the humanitarian search and rescue that is widely used in many emergency operations. Some tele-operated robotic SAR systems include the use of tethered mobile robots that can go deeply in the rubble to find victims. Moreover, a distributed wireless sensor network can be used to track SAR robots and can be used to acquire data to simulate parameters to be used in the future searching [2]. 


\subsection{Unmanned Aerial Vehicles}

Unmanned Aerial Vehicles can be classified into two main top-level configurations, fixed-wing and rotary-wings, where both kinds of UAVs have many benefits with few challenges regarding the control and guidance systems [3]. UAVs can be piloted or flown in autonomous mode. In autonomous mode, usually a pilot will assist with take off and landing of the vehicle, for safety reasons, and switch to autonomous mode in between these two events. To be useful for data collections, UAVs carry payloads that allow them to address different situations as needed. Four main types of sensor payloads that are carried by UAVs include 1) speed and distance, 2) infared and thermal, 3) image, and 4) chemical.

UAVs carrying various sensors provide a valuable solution for exploring our environment and an optimal way to collect information for a large number of applications. For example, companies and researchers use the photogrammetric products from UAVs in many fields such as agriculture, forestry, archaeology, environmental, emergency management, and traffic monitoring. Creating a 3-D map or digital surface model of an area of interest requires the vehicle to fly in a path such that the imaging sensors cover all the desired area.

When utilizing UAVs, coverage path planning must be considered. Coverage path planning means finding the route which covers every single point in the area for different shapes of the areas of interest. For example, the area could be any shape concave or convex polygons, circular, or any other shape 
imaginable.

\subsection{Unmanned Aerial Vehicle for SAR}

Over the last 20 years, UAVs (or drones) have become popular for many different applications, such as field surveillance, disaster and crisis management, and agriculture. For example, in 2006 after Hurricane Katrina, two UAVs helped the rescue team to survey the damaged area and locate people who were still in the wrecked buildings and facilities[4]. The current level of UAV accuracy allows this technology to be applied to many different type of complex tasks. UAVs can be used when there is a high risk to a vehicle operator or it is cost or time prohibitive to have a manned operation. Using UAVs also enable a rescue team to reach areas that can not be reached easily any other way. UAVs are controlled by a human from the ground control station and advanced techniques can be employed to deal with complex tasks.

SAR operations can minimize the time to find victims or missing persons by utilizing autonomous unmanned aerial vehicles (UAVs) to survey the environment and collect the necessary information about the position of the missing person or victim. Many factors need to be taken into account when developing a UAV algorithm for SAR. A few of the factors that should be considered are 1) the accuracy of data collected by the UAV, 2) the energy limit of each UAV, and 3) the hazards that are caused by different environmental factors. During SAR operations, time is very critical since any uncounted and unexpected delay can cause dramatic consequences that could mean loss 
of life. To provide appropriate critical support during the rescue operation in an unfriendly environment, it is beneficial to utilize UAVs. UAVs have many benefits for SAR, but the most significant ones are that they help the rescue team minimize the time and avoid dangerous areas during the operation.

If UAVs are to be used effectively for SAR, there must be efficient mission planning algorithms developed. Missions planning problems(MPPs) are considered a big challenge and NP-hard optimization problems. Classic planners use graph search or logic engines, but these type of planners have high computational cost to enable their algorithms to solve the problem to assist with completion of the mission. There is also another challenge with MPPs, which is the quality of the solution can be defined by using different parameters, such as fuel consumption and the cost of the mission. In these cases, a pareto-optimal frontier (POF) is calculated in such a way that we get the best solutions optimizing different goals at the same time. The concept of cooperation is considered one of the essential tools in mission planning, which occurs at a higher level when various UAVs work together in the same mission while they share data and take control together [5]. Cooperation algorithms can require unrealistic computational time, unless they are designed properly.

\subsection{Genetic Algorithms for Optimization}

Genetic Algorithms (GAs) are considered one of the most powerful techniques for optimization. They are stochastic algorithms whose search meth- 
ods model natural phenomena, survival of the fittest [6]. To design any GA which solves a given problem, we first need to model the problem's solutions utilizing chromosomes, usually a string of numbers. The chromosomes are then evaluated by using a fitness function that reflects the constraints of the problem and distinguishes between the fitness of the solutions. The fitness function can also be described as the measure of profit, utility, or goodness that we want to maximize. To continue to find better solutions, the GA utilizes three important processes, reproduction, crossover, and mutation to further refine the initial set of chromosomes developed.

Reproduction is the technique such that individual chromosomes (strings of numbers) are created. Crossover simply means that two members (parents) of the newly reproduced strings are mated randomly and new offspring chromosomes are created with attributes from each parent chromosome. Simply, mutation means the change of one gene in a chromosome to continue to try to get an optimal solution for the problem. The new population that follows from reproduction, cross over and mutation needs to be examined, the process of testing the new population starts with finding the fitness function values of the new chromosomes [7].

GAs have been utilized in a large number of fields, such as in system identification, robotics planning and scheduling, image processing, pattern recognition, and speech recognition. In general GAs have been used to optimize problems in applications like wire routing, scheduling, game playing, cognitive modeling, transportation, and database query [6]. For example, a 
GA planning scheme can used to provide an optimized trajectory to minimize space and time. In previous work, a GA was used to find the optimal planar robot manipulator trajectory where the goal was is to minimize the trajectory space/time without passing the maximum pre-defined torque by using direct kinematics to avoid the singularities. GAs were employed to optimize the point-to-point trajectory planning to for 3-link robot arm. The objective function in this case attempted to minimize the traveling time and space, while taking into account the other constraints of the system. For this application, quadrinomial and quintic polynomials were utilized to describe the segments that connect the initial, intermediate, and final points of the arm joint space. GAs have another important benefit, they are used to develop MATLAB program which generates an optimal shortest distance path plan for the mobile robot to cover the customized way points without colliding with the obstacles [8].

GAs have been used for UAV mission planning for many years [10]. Combined with space partitioning methods, GAs can assist in finding an optimized path to meet mission requirement and cover the desired area [11]. An example of previous work [9], GAs were used to determine an optimal path that would provide the required overlap of the area to generate a 3-D map of an area with special attention given to structures within the area [5]. For this previous work, a multiroter UAV was used and the GA assisted in finding the path the UAV was to fly [12]. The final flight path for a multirotor included way points, altitude, heading, and camera angle for all points along the path. 
The result of the flight was the gathering of all images needed to create 3-D map of the area with high resolution models of the structures within the area of interest. This work resulted in a patented algorithm, "Unmanned aerial vehicle 3D mapping system," USPatent 9618934.

\section{Research Methods}

The main goal of the research was to design a GA that will create a path for a UAV to search a circular area of interest, which may include some areas of avoidance where the UAV cannot fly. The GA was tested to determine which values of its parameters allow it to work better and more quickly. The research methods that will be discussed in this section include setting up the scenario of interest, determining the search area, and designing the GA to model the problem. The GA design includes, encoding the flight path as a chromosome, developing a crossover and mutation technique, and creating a fitness function that optimizes the flight. The following sections will elaborate on the process.

\subsection{Scenario of Interest}

In our research we will apply UAVs to SAR for a specific scenario. The problem we would like to address is finding the vulnerable missing person who has wandered of in a specific area of interest. For example, suppose that there is a mother and child going to a park. When they arrive the child 
starts playing with the other children, after 30 minutes the mother figures out that her child is not inside the park. Another case study, involves Mr Brown, age 75 years old, who had left the place where he is staying. He has been diagnosed with Alzheimer's and he has forgotten to take his cellphone,

so he can not been contacted. After an hour his caretaker starts to worry and needs assistance locating him. How can we effectively and efficiently search for these people?

Our research has designed a GA to determine the optimal path for a UAV to cover the region where the person may be found. A multiroter UAV will be utilized and the UAV will carry a video camera that can send back video

feed and images to a team on the ground. We did not address the image processing issues of this problem in our research, but focused on the optimal path planning of the UAV for coverage of the prospective area.

\subsection{Determining Search Area}

Considering that a person can walk in any direction, we decided to consider a circular area of interest. We will also need to take into the account that there could be tall vegetation, and buildings, and other areas that need to be avoided. 


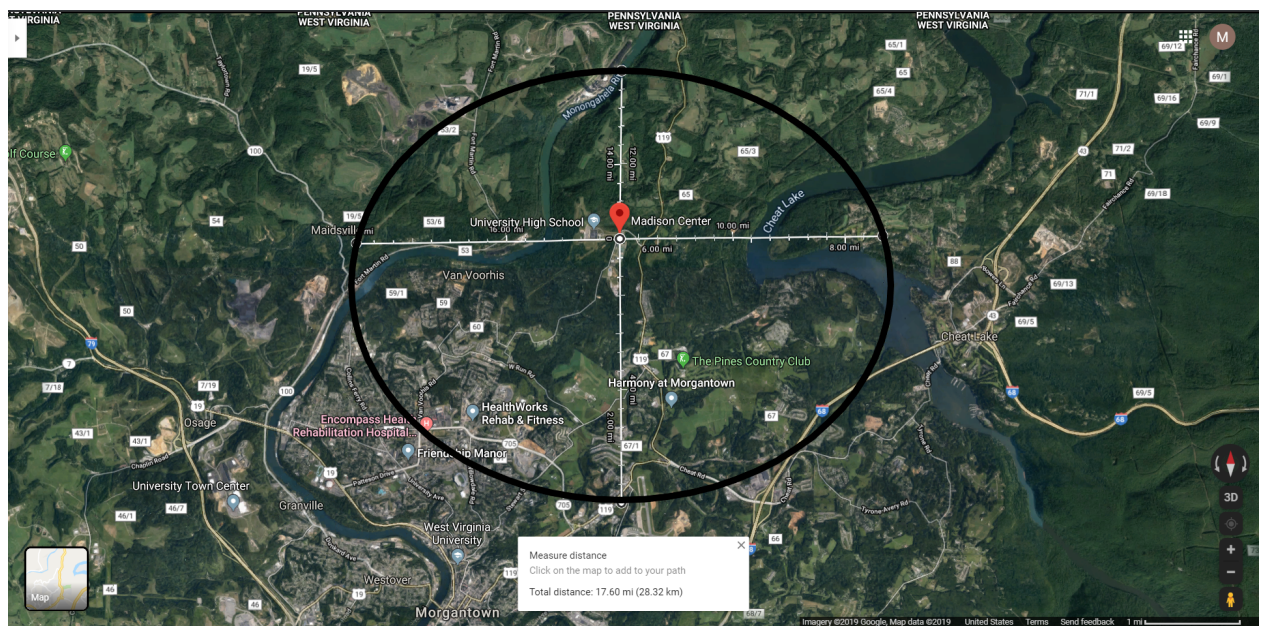

Figure 1: This figure shows a map with a circle where the UAV will search

In each case, we must consider how fast the person can walk, if there are any barriers they cannot cross, what habits they may have that would influence their path, etc. In the case of the child who may have wandered off out of the park, the child's rate of walking or running can be calculated to determine how far he/she might have gotten. In the second case study, Mr. Brown's therapy team, who are studying his behavior, estimate that based on his age and mental condition, Mr Brown can walk between 2.7 to 2.9 miles per hour. Figure 1 shows an example of the area with a circular region depicting were a person may be found.

This idea leads to a formula for calculating the area of the region to be searched, before any avoidance areas are deleted.

$$
A=\pi(R \times T)^{2}
$$


Where $A$ is area to be searched, $R$ is person's rate, and $T$ is time the person has been missing.

Once the area has been determine, a grid is overlaid onto the area. The grid is determined by the field of view of the camera being used. The center of each grid square will become a way point from which the UAV will fly. In general, to find the size of grid cells, $l \times l$, several things have to be taken into the account. First,the altitude of the UAVs, $h$ in feet, and the field of view of the camera, $f$ in degrees. The calculations can be done by

$$
l=h * \tan (f / 2)
$$

After the grid has been imposed on the circular region, then areas of avoidance can be subtracted by taking away grid cells. To begin with, we will utilize one UAV to search the area of interest, realizing if the area gets too large to search in a UAVs power supply capability, then we may need to find a plan to efficiently split the work between a team of UAVs. The method for this can be discussed later.

\subsection{Creating the GA for Path Planning}

We will use the GA in our work to find the optimal path planning for the missing person. In order to use GA for our area of interest, first we have to create GA to fit a particular problem and to do that we have to encode individual solution for the problem as a "chromosome." Each chromosome is 
a feasible solutions to the problem, that is a path the UAV can fly. However, we need to determine if the chromosome, candidate path, is optimal.

The chromosome for this application will be a list of number $1-4$, where each number represents a move of the UAV.

- 1 is LEFT

- 2 is UP

- 3 is RIGHT

- 4 is DOWN

The length of the list will be the number of grid square the UAV needs to visit. The following is an example of a chromosome, and the path of the UAV on the grid is depicted in Figure 2.

Sample Chromosome:

11122233333444433322211111114444443333

If this is a chromosome, then starting in the middle of the grid, the UAV would make the following moves Each time it moves the square it occupies becomes its new start position.

Left, Left, Left, Up, Up, Up, Right, Right, Right, Right, Right, Right, Down, Down, Down, Down, Right, Right, Right, Up, Up, Up. Left, Left, Left, Left, Left, Left, Left, Down, Down, Down, Down, Down, Down, Right, Right, Right, Right

We start the GA process by generating a list of 100 chromosome (the initial population). 


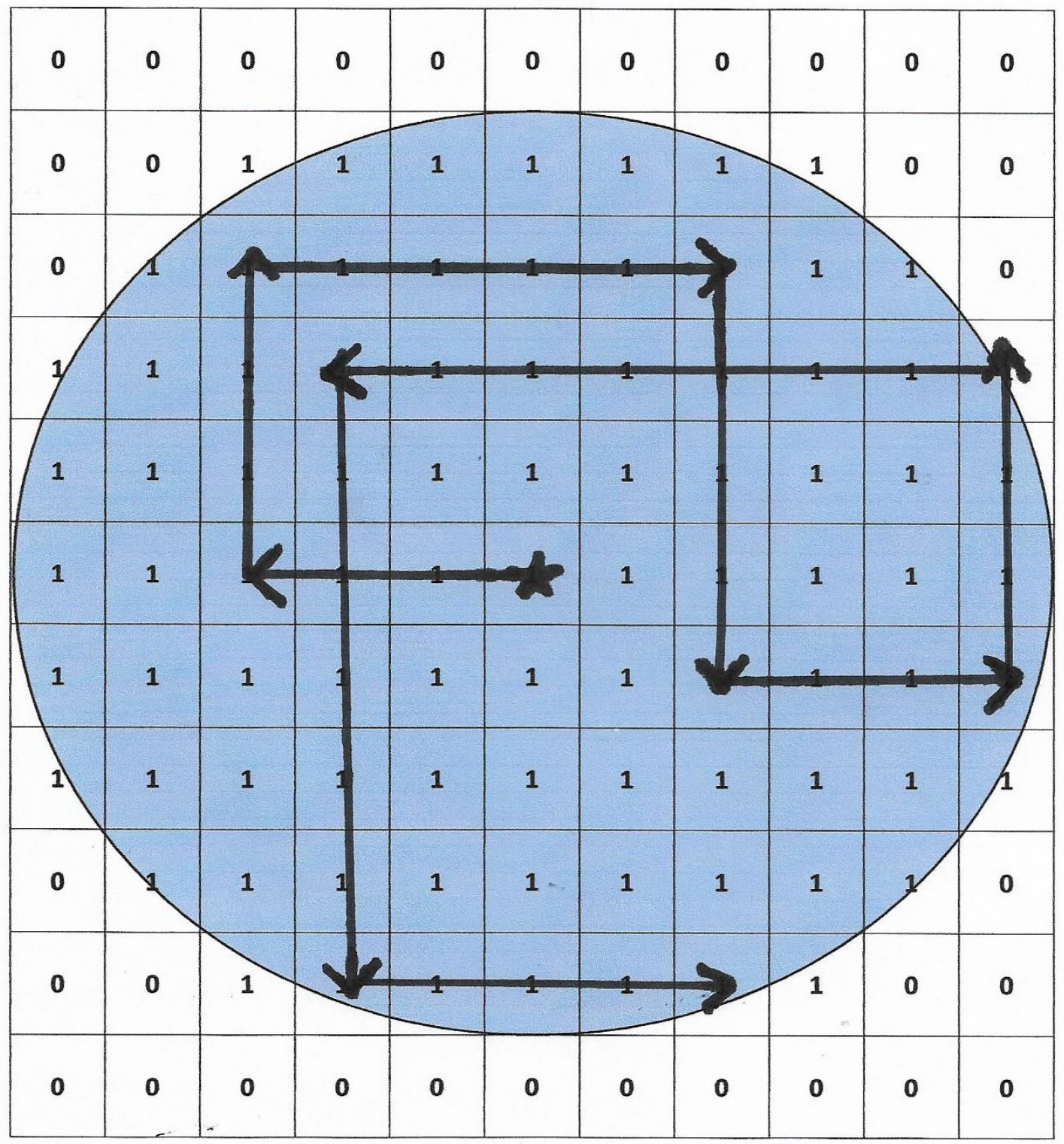

Figure 2: Example Grid, 0 represents the areas that are outside the target area and 1 represents areas that should be visited. The black line shows how the UAV can move. 


\subsubsection{Fitness Function}

The fitness function is customized for each problem to be solved. In our case the fitness function is a fraction that consists of the number of open grid cells covered by the UAV when following the flight path outline by chromosome $\mathrm{c}$ is in the numerator and total number of open grid cell is located in the denominator.

$$
F(c)=\left(N_{I} / N(c)\right) \times 10^{-\left(N_{I}-N(c)\right)}
$$

Where $F(c)$ is the fitness of chromosome $c, N(c)$ is the number of grid squares covered by the chromosome, and $N_{I}$ is total number of open grid cells that need to be covered to complete the search. The $10^{-\left(N_{I}-N(c)\right)}$ penalizes chromosomes that have a long path that backtracks and covers the same grid squares several times (path too long). It also penalizes chromosomes that do not cover all the grid cells (path too short). The fitness value of 1 would represent a perfect flight path, with no overlapping in the path or grid squares left uncovered.

The closer the $F(c)$ is to 1 the better the fitness of the chromosome. A fitness value greater than 1 indicates that the path is longer than the ideal path. This means that backtracking occurred. A fitness function less than 1 indicates that there were open cells not visited. 


\subsubsection{Crossover, Mutation, and Elitism}

The GA then uses crossover and mutation to develop better solutions from the initial populations. After the crossover and mutation each successful generation is better than one before where successful generation means the generation that is usually tested by the fitness function and its value is very close to 1 .

The initial population is a hundred chromosomes. The algorithm uses the Roulette wheel method to select chromosomes for mating by utilizing the chromosomes with the top fitness as the first parent. The second parent is picked randomly from the other chromosomes. A single point crossover is then performed by selecting a single random number between 1 and the

length of chromosome. Next, the entries from 1 to that number from the first parent are appended to the entries from the random number to length from the other parent.

We mutate by choosing $30 \%$ of the children from the new generation, then from those selected, $10 \%$ of the entries of the chromosome were mutated by changing to another digit from 1 to 4 . Mutation happens every generation.

In order for the best solution from each generation to continue to increase, or at least never decrease, we keep the chromosome with the highest fitness and put it into the next generation. This process is called Elitism and will ensure the solution will be monotonically increasing.

Then the children that result from the crossover and mutation and the top performer are outputted as the new generation and the process start over 
again.

\section{Results}

We developed a plan to test the GA in order to see what values of the parameters produced the best results. The following parameters can be changed:

- Size of Population (Pop) - This is the number of chromosomes in the initial and subsequent generations.

- Number of Generations (Gen) - This is the number of different populations that will be developed.

- Error (Err) - this is the amount the chromosome is allowed to grow past the ideal length that would cover all open grid cells. An error is necessary to accommodate the need to backtrack or visit one cell more than one time.

- Mutation Rate per Population (MutPop) - This in percentage of chromosomes in the population that will be mutated in any one generation.

- Mutation Rate per Chromosome (MutChr) - This is the percentage of chromosome entries that will be changed during any mutation.

In order to test each parameter, we will hold the others constant and run a test changing only one parameter at a time. When we find the best value 
for each parameter independently, then we will run the GA with all the best values at once.

Example 1: A 24 by 24 circular area was created and a 2 by 2 square avoidance area was positioned in the top left part of the circle (cells $(8,7)$, $(8,8),(9,7),(9,8))$

Each parameter was tested using three different values to see what value would yield the best results. Each value of the parameter was run three times to determine the best of the three runs. The tables, graphs and diagrams in Figures 3 through 8 below depict the best fitness for each parameter. The table shows the values that were tested. The graph shows the best fitness value of each generation plotted against the number of the generation. The diagram depicts the coverage of the path given the best chromosome for that test. Any red square or dark blue squares are not covered. The red square that appears in the top left of the circle is put there purposely as an area of avoidance (a place that the UAV may not visit). 


\begin{tabular}{|l|l|l|l|l|l|}
\hline $\begin{array}{l}\text { Parameters/ } \\
\text { Values }\end{array}$ & Pop & Gen & Err & MutPop & MutChr \\
\hline & $\mathbf{1 0 0}$ & 3000 & 1.4 & .3 & .1 \\
\hline & $\mathbf{2 0 0}$ & 3000 & 1.4 & .3 & .1 \\
\hline & $\mathbf{3 0 0}$ & 3000 & 1.4 & .3 & .1 \\
\hline
\end{tabular}
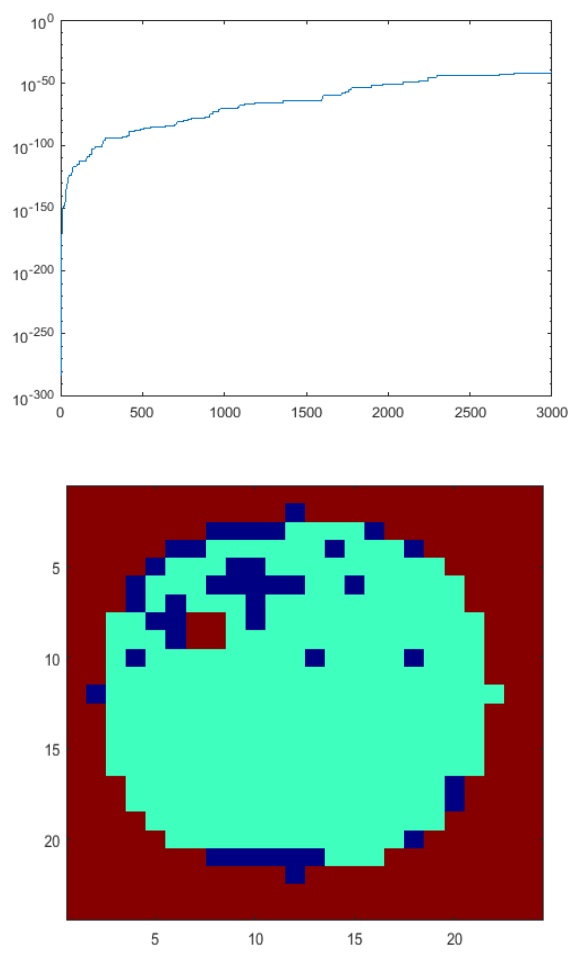

Figure 3: Change for Population Size. The graph depicts the population size of 200 that was found to give the best results. 


\begin{tabular}{|l|l|l|l|l|l|}
\hline $\begin{array}{l}\text { Parameters } \\
\text { /Values }\end{array}$ & Pop & Gen & Err & MutPop & MutChr \\
\hline & 100 & $\mathbf{3 0 0 0}$ & 1.4 & .3 & .1 \\
\hline & 100 & $\mathbf{4 0 0 0}$ & 1.4 & .3 & .1 \\
\hline & 100 & $\mathbf{5 0 0 0}$ & 1.4 & .3 & .1 \\
\hline
\end{tabular}
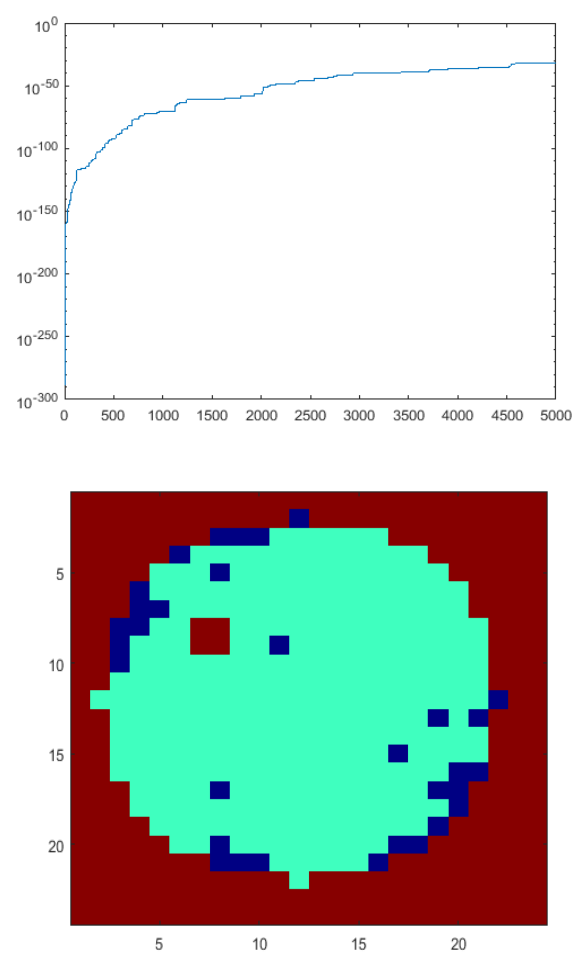

Figure 4: Change for Number of Generations. The graph depicts the number of generation 5000 that was found to give the best results. 


\begin{tabular}{|c|c|c|c|c|c|}
\hline $\begin{array}{c}\text { Parameters } \\
\text { /Values }\end{array}$ & Pop & Gen & Err & MutPop & MutChr \\
\hline & 100 & 3000 & $\mathbf{1 . 4}$ & .3 & .1 \\
\hline & 100 & 3000 & $\mathbf{1 . 2}$ & .3 & .1 \\
\hline & 100 & 3000 & $\mathbf{1 . 6}$ & .3 & .1 \\
\hline
\end{tabular}
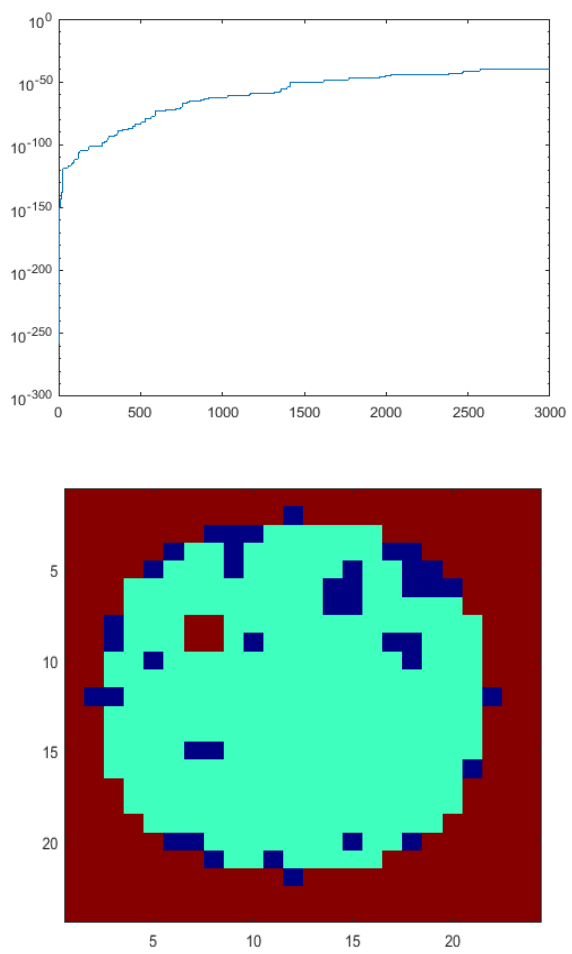

Figure 5: Change for Error. The graph depicts the error of 1.2 that was found to give the best results. 


\begin{tabular}{|l|l|l|l|l|l|}
\hline $\begin{array}{l}\text { Parameters } \\
\text { /Values }\end{array}$ & Pop & Gen & Err & MutPop & MutChr \\
\hline & 100 & 3000 & 1.4 &. $\mathbf{3}$ & .1 \\
\hline & 100 & 3000 & 1.4 & .2 & .1 \\
\hline & 100 & 3000 & 1.4 &. $\mathbf{4}$ & .1 \\
\hline
\end{tabular}
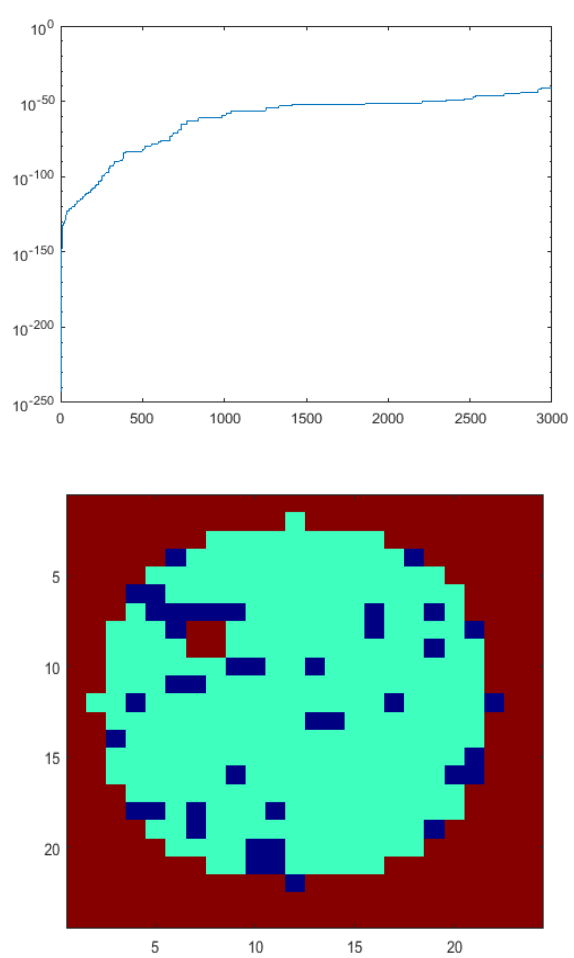

Figure 6: Change for Population Mutation Rate. The graph depicts the population mutation rate of .2 that was found to give the best results. 


\begin{tabular}{|l|l|l|l|l|l|}
\hline $\begin{array}{l}\text { Parameters } \\
\text { Values }\end{array}$ & Pop & Gen & Err & MutPop & MutChr \\
\hline & 100 & 3000 & 1.4 & .3 &. $\mathbf{1}$ \\
\hline & 100 & 3000 & 1.4 & .3 & $\mathbf{. 2}$ \\
\hline & 100 & 3000 & 1.4 & .3 & $\mathbf{. 3}$ \\
\hline
\end{tabular}
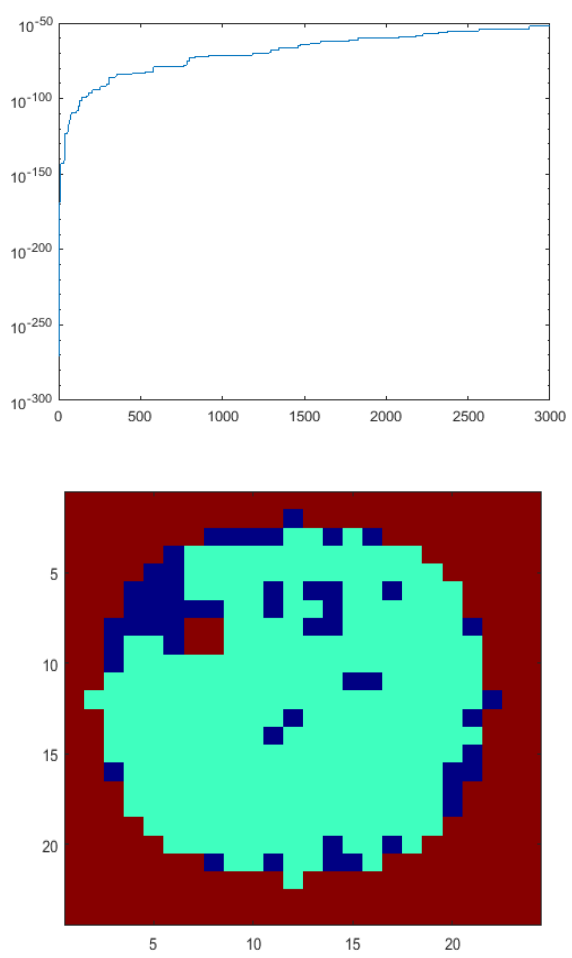

Figure 7: Change for Chromosome Mutation Rate. The graph depicts the chromosome mutation rate of 0.1 that was found to give the best results. 


\begin{tabular}{|c|c|c|c|c|c|}
\hline $\begin{array}{c}\text { Parameters/ } \\
\text { Values }\end{array}$ & Pop & Gen & Err & MutPop & MutChr \\
\hline & 200 & 5000 & 1.2 & 2 & 1 \\
\hline & & & & & \\
\hline
\end{tabular}
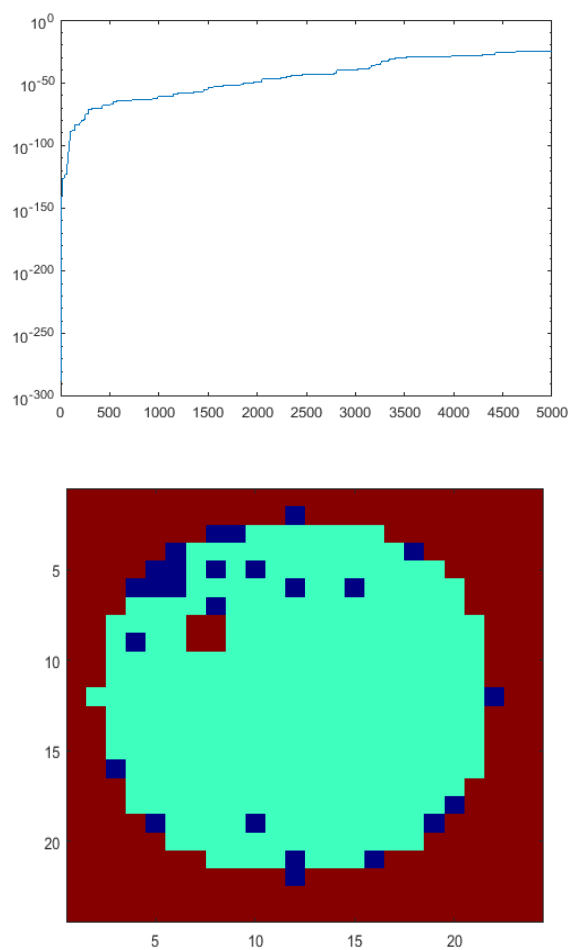

Figure 8: Finally, we ran the best of each of the parameters together. The graph depicts the results of the individual best parameters used at the same time. 
Example 2: In this example it is assumed a large L-shaped building is present in the top left of the circle and there is a river that cannot be crossed, cutting of the top right part of the circles. A 24 by 24 circular area was created and an L-shaped avoidance area was positioned in the top left part of the circle (cells $(8,7),(8,8),(9,7),(9,8),(10,7),(10,8),(10,9),(10,10)$, $(11,7),(11,8),(11,9),(11,10))$ Also, an area of avoidance was positioned in the top right of the circle angling from $(1,13)$ on a diagonal down to $(8,20)$ and all cells above that diagonal were designated as an area of avoidance. Figure 9 shows this example and the values of the parameters used for the run. 


\begin{tabular}{|c|c|c|c|c|c|}
\hline $\begin{array}{c}\text { Parameters/ } \\
\text { Values }\end{array}$ & Pop & Gen & Err & MutPop & MutChr \\
\hline & 200 & 5000 & 1.2 & .2 & .1 \\
\hline & & & & & \\
\hline
\end{tabular}
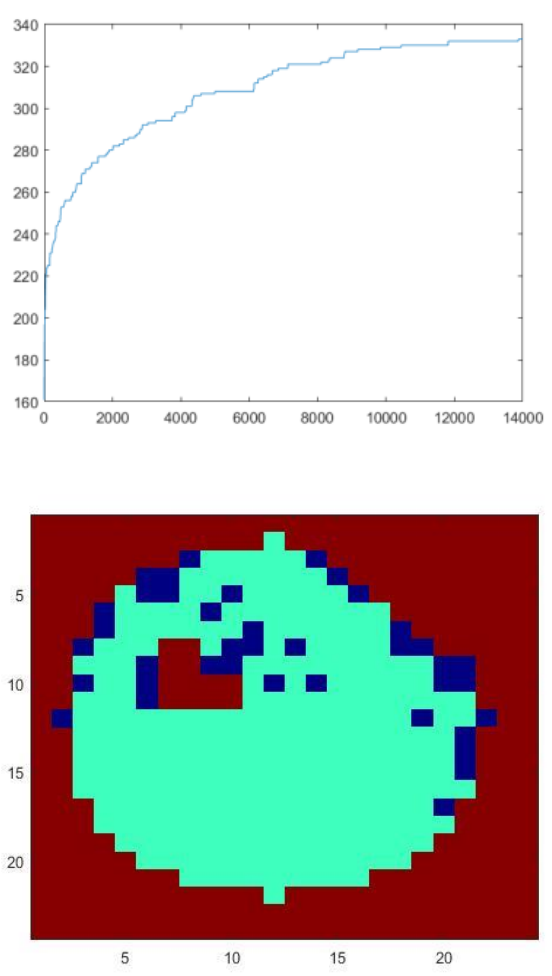

Figure 9: The areas of avoidance were changed and then we ran the best of each of the parameters together. The graph depicts the results of the individual best parameters used at the same time. 


\section{Conclusion and Future Work}

This research focused on searching for persons from vulnerable populations and considering how they might travel and what type of area would need to be searched. A GA was designed and implemented in Matlab (code in Appendix) for path planning of an Unmanned Aerial Vehicle (UAV) to search for the lost person in the area of interest. The GA was tested with different values for parameters to choose the best ones that would cover more of the area of interest. Two different types of areas were investigated to show that the GA is versatile and can develop paths for diverse scenarios with various areas of avoidance that exclude portions of the circular area.

Since time is very important when a person is missing the algorithm should run as fast as possible and provide the best solution possible in a short time. In future work, the algorithm could be modified to run larger numbers of generations in a shorter time. Even though the algorithm can now find a good solution in several second, there were still grid cell that were not visited. Since all grid cells need to be visited it would take a much longer time to find such a solution. There might be ways to modify the construction of the chromosomes of the fitness function that would speed up the process. Depending on how long a person has been missing, the area of interest could grow very large. Depending on the vehicles available, small UAVs often have a short battery life (20-30 minutes). So in future work it would be good to investigate how to divide the area efficiently between multiple UAVs. This 
can be done by determining the appropriate number of UAVs needed, and then equitably dividing the area between the vehicles. After this is done, the GA can be used to develop paths for each of the UAVs.

This research provides a proof of concept and a basis for further study in the area of using a GA for path planning a UAV for search and rescue of persons from vulnerable populations. 


\section{References}

[1] Vikki Fenton, "The use of dogs in search, rescue and recovery," Journal of Wilderness Medicine, ISSN 0953-9859, (1992): 292-300.

[2] Albert Ko and Henry Y.K. Lau, "Robot Assisted Emergency Search and Rescue System With a Wireless Sensor Network," International Journal of Advanced Science and Technology 2 (2009): 69-78.

[3] Tauã M. Cabreira, Lisane B. Brisolara, and Paulo R. Ferreira Jr., "Survey on Coverage Path Planning with Unmanned Aerial Vehicles," Drones 3 (2019).

[4] Sonia Waharte and Niki Trigoni, "Supporting Search and Rescue Operations with UAVs," International Symposiom on Robots and Security (2010).

[5] Bello-Orgaz Ramirez-Atencia and Camacho R-Moreno, "Solving complex multi-UAV mission planning problems using multi-objective genetic algorithms," Soft Computing 21 (2017): 4883-4900.

[6] Z. Michalewics, Genetic Algorithms + Data Structures Evolution Programs, (Springer-Verlag, 1992).

[7] David Goldberg, Genetic Algorithms in Search, Optimization and Machine Learning, (Reading, Mass: Addison Wesley Publishing Co, 2006). 
[8] T. Davies and A. Jnifene, "Multiple Waypoint Path Planning for a Mobile Robot using Genetic Algorithms," International Conference on Computational Intelligence for Measurement Systems and Applications, La Coruna (2006): 21-26.

[9] M. M. Trujillo, M. Darrah, K. Speransky, B. DeRoos, and M. Wathen. "Optimized flight path for 3D mapping of an area with structures using a multirotor," International Conference on Unmanned Aircraft Systems (ICUAS) (2016): 905-910.

[10] Marjorie Darrah, Edgar Fuller, Thilanka Munasinga, Kristin Duling, Mridul Gautam and Mitchell Wathen "Using Genetic Algorithms for Tasking Teams of Raven UAVs," Journal of Intelligent Robotic Systems 70 (2013): 361-371.

[11] Marjorie Darrah, William Niland, and Brian Stolarik, "UAV cooperative task assignments for a SEAD mission using genetic algorithms," AIAA Guidance, Navigation, and Control Conference and Exhibit 6456 (2006).

[12] Marjorie Darrah, Jay Wilhelm, Thilanka Munasinghe, Kristin Duling, Steve Yokum, Eric Sorton, Jonathan Rojas and Mitchell Wathen, "A Flexible Genetic Algorithm System for Multi-UAV Surveillance: Algorithm and Flight Testing," Unmanned Systems 3(1) (2015): 49-62. 


\section{Appendix}

\subsection{Code for GA}

height $=24 ; \%$ initializing the dimensions for the FlightSpace

length1 $=24$ :

num $=100 ; \%$ number of individuals in each generation.

generations $=3000$; $\%$ number of generations to run

error $=1.4 ; \%$ multiplier of the extra amount of moves you would like.

FlightSpace $=$ zeros (height, length1); \%Flight Space for UAV.

NewFlightSpace $=$ changeFlightSpace (height, length1, FlightSpace);

$\%$ This function changes the shape of the flight space.

IdealFitness $=$ countZeros(height, length1, NewFlightSpace);

$\%$ Calculating the Ideal Fitness.

$\%$ Done by counting the total flyable space (num of 0 s).

initialpop = makePop(error, num, (IdealFitness) $)$;

\%aking the Population

pop = initialpop;

yaxis $=[]$; \% to hold all of the number of squares covered per generation.

for $j=1$ :generations

$\%$ Fitness function

$\operatorname{len}=\operatorname{length}(\operatorname{pop}(1,:))$;

$\mathrm{y}=0$;

for $i=1: \operatorname{length}(\operatorname{pop}(:, 1))$ 
$\mathrm{y}=$ FitnessForDarrah(NewFlightSpace, IdealFitness,

$\operatorname{pop}(i,:))$;

$\operatorname{pop}(i, l e n+1)=y$;

end

temppop $=$ pop; \%this section makes the new generation

$[$ Ten, BotNinety $]=\operatorname{TopTen}(\mathrm{pop})$;

[NewGen, top] = Crossover (Ten, BotNinety, num);

$\operatorname{yaxis}(j)=y ; \% \operatorname{disp}($ words $)$

pop $=$ NewGen;

end

words $=[$ "The Top performer in Gen ", j, " covers ",

NumOfGridsCovered (NewFlightSpace,top)] ; \%disp (words)

BestPath $=\operatorname{showPath}($ NewFlightSpace, top);

PrepBestPath $=$ prepForColor (height, length1,

BestPath);

image (PrepBestPath);

colorMap $=$ jet $(256)$;

colormap (colorMap); \%xlabel ('Red = Unexplorable, Blue = Uncovered land, green = C

figure() \%xlabel("Generations") \%ylabel("Number of Grids covered")

semilogy (yaxis); 


\subsection{Crossover Code}

function $[X$, top $]=$ Crossover (Ten, BotNinety, num)

$\%$ Crossover of the top ten

$\%$ Output is the new 100 individuals + the best one from the previous gen.

$\mathrm{X}=[]$;

len $=\operatorname{length}(\operatorname{Ten}(1,:))$;

SumOfFitness $=\operatorname{sum}(\operatorname{Ten}(:$, len $))$;

top $=\operatorname{Ten}(1,1:$ len-1);

top1 = Ten $(1,1:$ len $)$;

for $i=1: 10$

$\operatorname{Ten}(i$, len +1$)=\operatorname{Ten}(i$, len $) /$ SumOfFitness;

end

probabilities $=$ Ten $(:$, len +1$) ; \%$ array of the probabilities

len1 = length (probabilities);

for $i=1:$ num

if ( isempty(find(probabilities<1, 1))) \%Line 18-55 is the pinwheel

style of picking the first parent

if $(\min ($ probabilities $) \sim 0)$

probabilities $=1 / \mathrm{min}$ (probabilities) $*$ probabilities;

else

temp = probabilities;

temp $($ probabilities $==0)=$ inf ;

probabilities $=1 / \mathrm{min}($ temp $) *$ probabilities; 


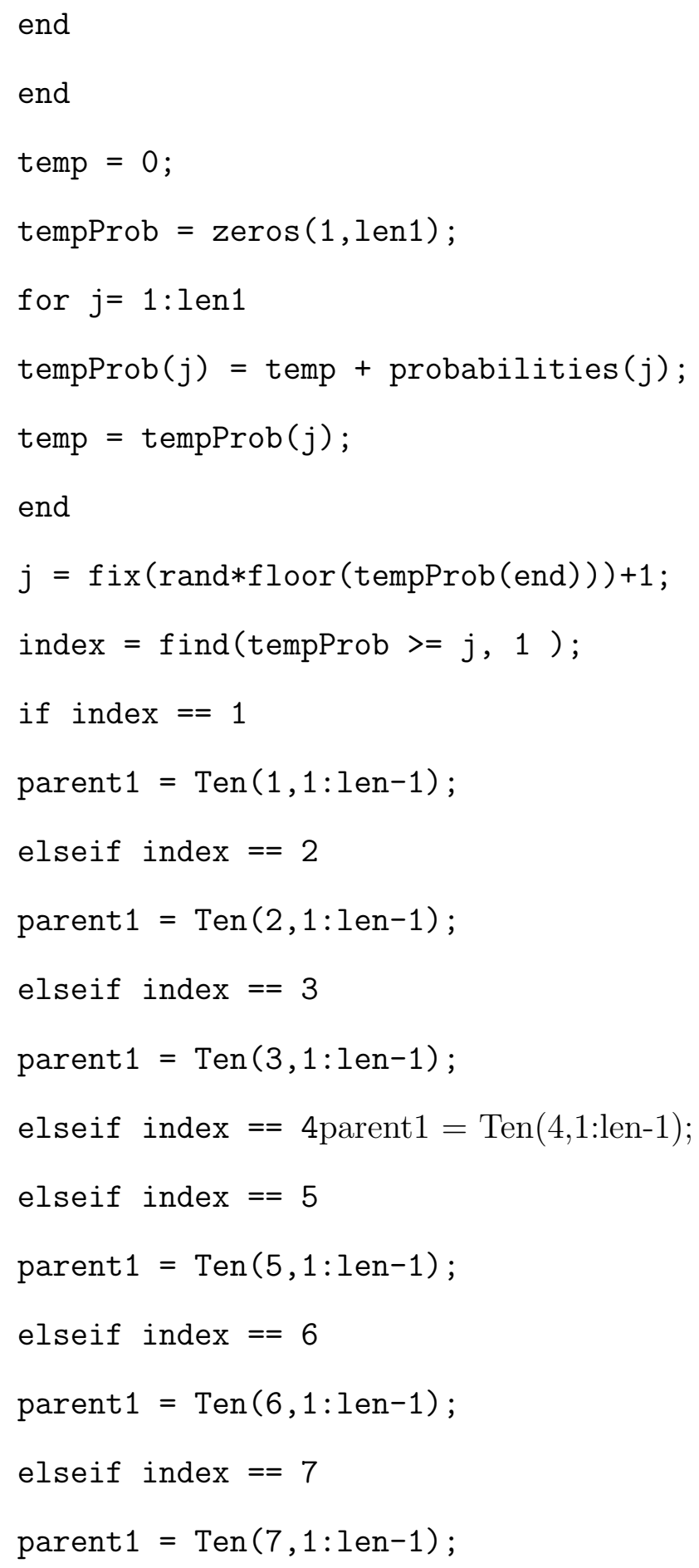




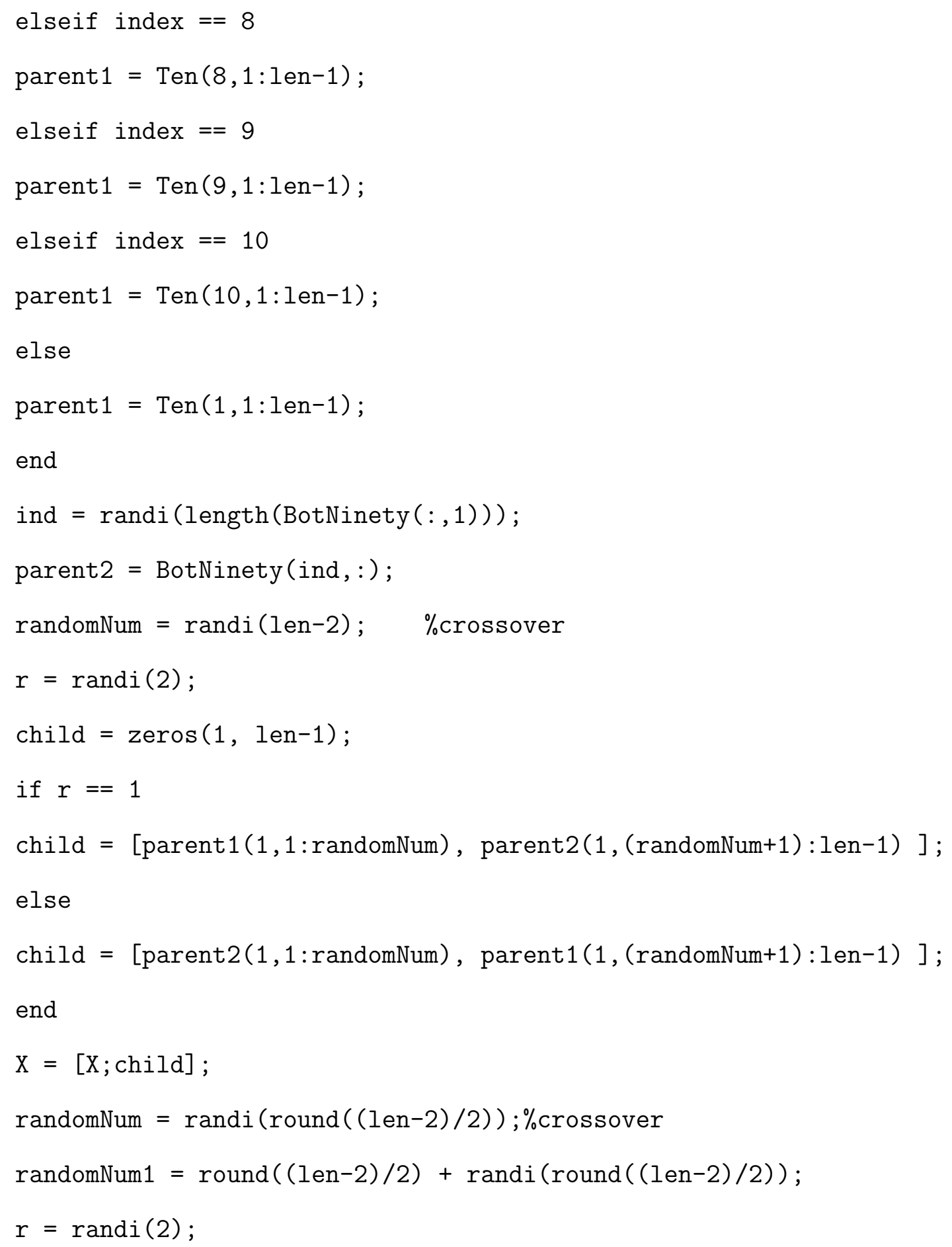




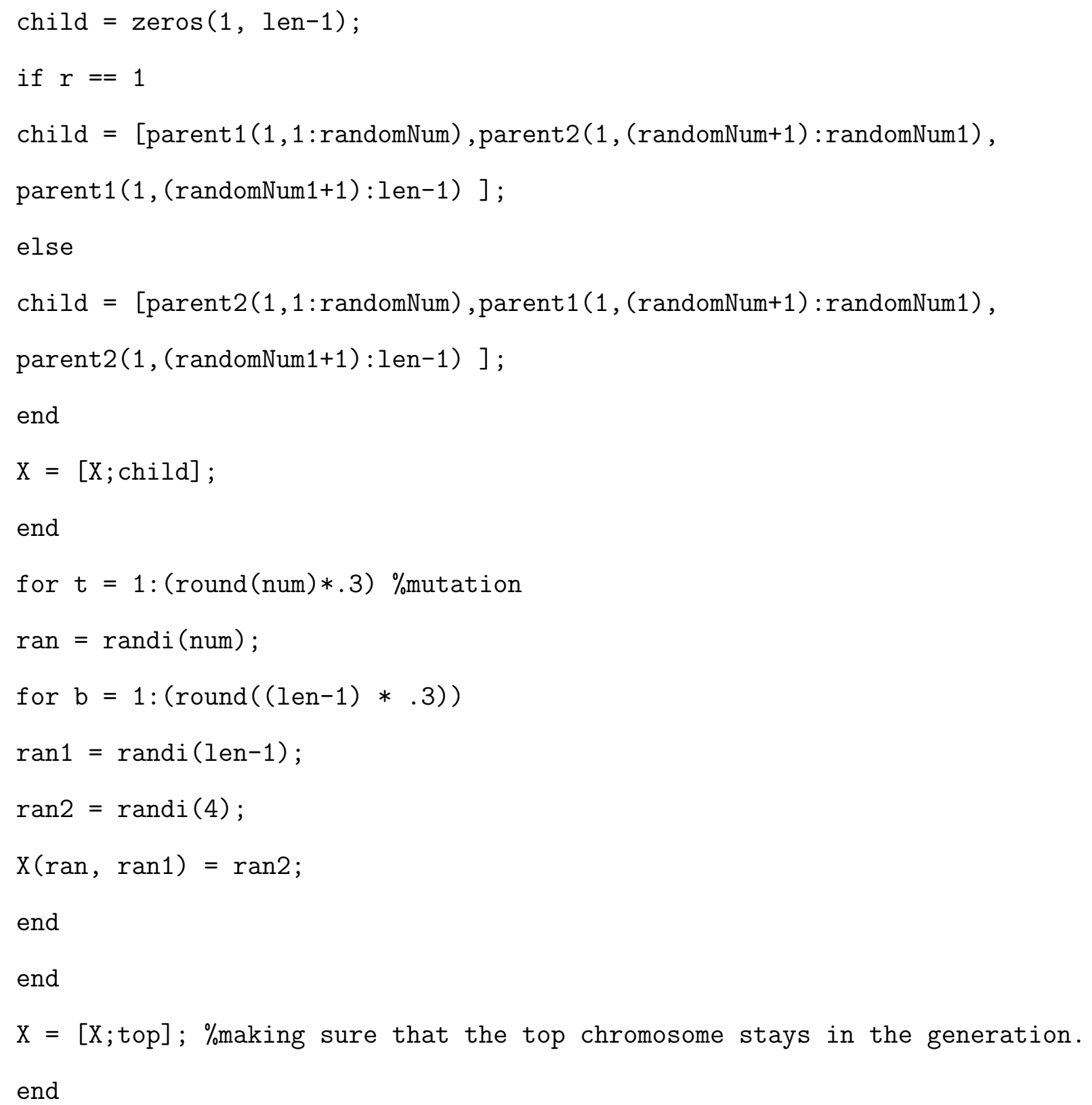

